
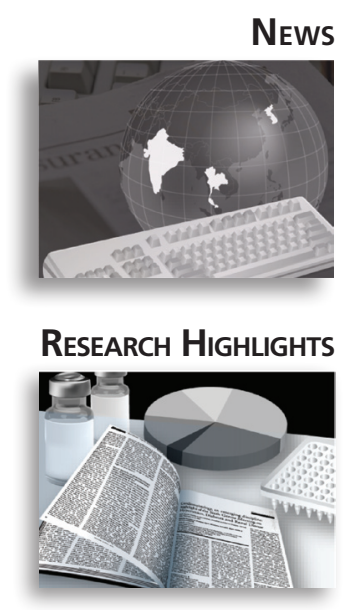

Conference Scene

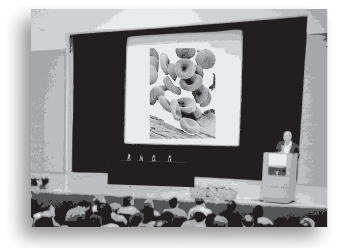

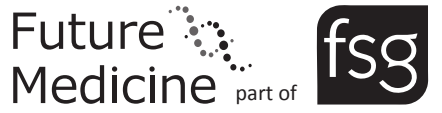

\section{Medical diagnostics welcomes} promising new ultrasensitive biosensor

The Flexure-FET biosensor could open up opportunities for early detection of
cancer and personalized medicine.

Researchers at Purdue University (IN, USA) have created a new ultrasensitive biosensor. The device, called a Flexure sensitive Field Effect Transistor (Flexure-FET biosensor), holds promise for personalized medicine tailored to the specific biochemistry of individual patients and potential in the early detection of cancer.

The research group comprises graduate student Ankit Jain; former doctoral student who is now a faculty member at the Indian Institute of Technology (Mumbai, India), Pradeep Nair and Purdue University Professor of Electrical and Computer Engineering, Muhammad A Alam. The findings are published in the June issue of the Proceedings of the National Academy of Sciences of the USA.

\section{"You can make the device sensitive to almost any molecule as long as you configure the sensor properly ... a key innovation is that this is achieved without the need for a reference electrode, or additional sophisticated instruments."}

The researchers state that the FlexureFET biosensor could achieve exponentially high sensitivity compared to other biosensors, as it combines attributes of two distinctly different sensor types: a mechanical and electrical; thereby, overcoming the respective limitations of classical electrical and mechanical nanoscale biosensors.

The proposed uses of a Flexure-FET biosensor could be several hundred times more sensitive than other biosensors, claims Muhammad Alam: "Individually, both of these types of biosensors have limited sensitivity, but when you combine the two, you get something that is better than either."

"Eliminating the need for a reference electrode enables miniaturization and makes it feasible for low-cost, point-of-care applications in doctors' offices..."

The device combines a mechanical sensor (vibrating silicon cantilever), which identifies a biomolecule based on its mass or size, with an electrical sensor (a transistor located under the cantilever) that identifies molecules based on their electrical charge. The new sensor detects both charged and uncharged biomolecules, allowing a broader range of applications than either type of sensor alone.

Alam explains, "You can make the device sensitive to almost any molecule as long as you configure the sensor properly."

Furthermore, a key innovation is that this is achieved without the need for a reference electrode, or additional sophisticated instruments.

"Eliminating the need for a reference electrode enables miniaturization and makes it feasible for low-cost, point-ofcare applications in doctors' offices," Alam explains.

The authors state that the high sensitivity of Flexure-FET can eliminate the need of PCR for the amplification of DNA and hence reduce the cost of sequencing.

The two potential applications of the device are personalized medicine, in which an inventory of proteins and DNA 
is recorded for individual patients to make more precise diagnostics and treatment decisions; and the early detection of cancer and other diseases.

In early cancer diagnostics, the sensor makes possible the detection of small quantities of DNA fragments and proteins deformed by cancer long before the disease is visible through imaging or other methods.

A US patent application has been filed for the concept behind the Flexure-FET biosensor, with emphasis placed on its distinctive nonlinear operation specifically optimized for ultrasensitive detection of biomolecules.

Sources: Jain A, Nair PR, Alam MA. Flexure-FET biosensor to break the fundamental sensitivity limits of nanobiosensors using nonlinear electro-mechanical coupling. Proc. Natl Acad. Sci. USA 109(24), 9304-9308 (2012); Press release: Ultrasensitive biosensor promising for medical diagnostics: www.purdue.edu/newsroom/rese arch/2012/120514AlamBiosensor.html

\section{Personalized medicine: access versus pay}

Research in both the private and public sector has identified a plethora of biomarkers suitable for diagnosing disease and assessing patients' responses to therapy. This research could lead to the development of efficient tests tailored to personalized medicine. However, alongside research developments, legal conflict has arisen, questioning whether a private company can own the rights to a particular biomarker.

To address this, Jason Karlawish, professor of medicine, medical ethics and health policy in the Perelman School of Medicine at the University of Pennsylvania (PA, USA) and co-author Aaron Kesselheim, from Brigham and Women's Hospital and Harvard Medical School (MA, USA) recently published a perspective piece in the New England Journal of Medicine, which discusses a series of high-profile court cases challenging the scope of patent protection policies and procedures.

A key argument championed by many is that without the relevant patents protecting biomarker medical discoveries, there will be a danger of the costs associated with biomarker discovery never being recovered.

Recently a US Supreme Court ruling unanimously "rendered invalid two patents covering a method for determining proper drug dosage," as reported in Nature. This has raised questions regarding who is responsible for paying for personalized medicine, and how.

For those patents that may succeed, the authors of the New England Journal of Medicine article question whether the law will be hindering the practice and research in the field by covering not only the use of the biomarkers, but also their use in diagnoses and further development.

\section{“...alongside research developments,} legal conflict has arisen questioning whether a private company can own the rights to a particular biomarker."

Justice Breyer from the Supreme Court went on to question whether innovation in diagnostic methods needs special market-exclusivity protection. However, the authors of the perspective suggest that enhanced public funding, public-private partnerships and open-source consortia may improve biomarker discovery and development, more so than a private model. They claim that, "the Supreme Court's move to free the fundamental processes of medical diagnosis from private ownership ... could ultimately enhance the public health."
The authors also consider that "a patentable process now needs to involve an inventive and novel application of a law of nature beyond well-understood, routine, conventional activity, previously engaged by those in the field."

\section{"A key argument championed by \\ many is that without the relevant patents protecting biomarker medical discoveries, there will be a danger of the costs associated with biomarker discovery never being recovered."}

Research into novel biomarkers capable of diagnosing diseases and tailoring treatment decisions is currently a major focus; therefore, there will be an ever-increasing need to find and direct solutions to balance the needs of those providing the technologies, strategies and tools with those of the patients who wish to access the information derived from such personalized medicine.

Sources: Kesselheim AS, Karlawish J. Biomarkers unbound - the Supreme Court's ruling on diagnostic-test patents. N. Engl. J. Med. doi:10.1056/ NEJMp1204164 (2012) (Epub ahead of print); Nature news: US Supreme Court upends diagnostics patents. doi:10.1038/nature.2012.10270 (2012): www.nature.com/news/us-supremecourt-upends-diagnostics-patents-1.10270.

\section{About the News \& Views}

The News \& Views highlights some of the most important events and research in the field of pharmacogenomics. If you have newsworthy information, please contact: Natalie Harrison, Managing Commissioning Editor, Pharmacogenomics, Future Medicine Ltd, Unitec House, 2 Albert Place, London, N3 1QB, UK; Tel.: +44 (0)20 8371 6090; Fax: +44 (0)20 8371 6099; n.harrison@futuremedicine.com 


\section{SLCO1B1 linked to commonly prescribed simvastatin and induced myopathy}

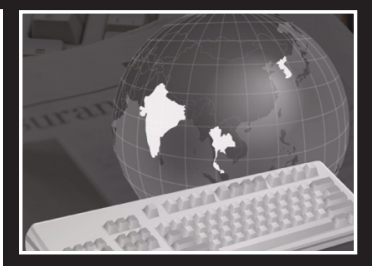

The Clinical Pharmacogenetics Implementation Consortium (CPIC) has recently published a clinical dosing guideline for SCLO1B1 genotype and simvastatin dosing in the journal Clinical Pharmacology of Therapeutics. The guideline explores the relationship between the rs4149056 SNP and clinical outcome for all statins; and represents the sixth publication from the CPIC.

The goal of the CPIC is to address some of the barriers facing the implementation of pharmacogenetic tests into clinical practice. The consortium provides guidelines that enable the translation of genetic laboratory test results into actionable prescribing decisions for specific drugs.

Statins are a commonly prescribed medication used to treat hypercholesterolemia and prevent cardiovascular diseases. The reduction in cholesterol by simvastatin therapy has been a great success of modern medicine. They function by inhibiting the rate-limiting enzyme, HMG-CoA reductase, involved in cholesterol formation, thereby lowering cholesterol levels. Statins are well tolerated in general; however, they can lead to myopathy with symptoms ranging from mild muscle pain to fatal rhabdomyolysis. The potential risk of myopathy is increased when the drugs are administered at higher doses and with certain other medications.

The recent guideline presents dosing recommendations for simvastatin only, since evidence linking risk of myopathy to a common nonsynonymous coding SNP, rs4149056 (c.521T>C, p.V174A, $\left.S L C O 1 B 1^{*} 5\right)$ in the SLCO1B1 gene has been shown to be significantly associated with marked increase in systemic exposure to simvastatin (than for any other statin).

The overall effect size and significance of this association with simvastatin had an odds ratio for myopathy of 4.5 (95\% CI: 2.6-7.7) per copy of the C allele and 16.7 (95\% CI: 4.7-61.1) among CC homozygotes as compared with the TT genotype.

Due to the nature of the association of SLCO1B1 and the risk of muscle toxicity, the guideline represents a valuable contribution to the clinical arena.

Sources: Wilke RA, Ramsey LB, Johnson SG et al. The Clinical Pharmacogenomics Implementation Consortium: CPIC guideline for SLCO1B1 and simvastatin-induced myopathy. Clin. Pharmacol. Ther. doi:10.1038/clpt.2012.57 (2012) (Epub ahead of print); SEARCH Collaborative Group, Link E, Parish $S$ et al. SLCO1B1 variants and statin-induced myopathy - a genomewide study. N. Engl. J. Med. 359(8), 789-799 (2008).

\section{$V D R$ genetic variants associated with a wide variety of diseases and therapeutic responses}

Research led by Poon et al., including collaborators from the USA, Canada and Denmark, have recently written an updated Very Important Pharmacogene (VIP) Summary for the VDR gene published in the Journal Pharmacogenetics and Genomics. The paper describes the importance of vitamin $\mathrm{D}$ receptor (VDR) binding to the active form of vitamin D (1,25-dihydroxyvitamin $\mathrm{D}_{3}$ ) and focuses on clinically relevant associations between treatment responses in the presence of $V D R$ genetic polymorphisms and has already received some follow-up interest within the research community.

The human VDR protein is a transacting transcriptional factor, that by binding to the active form of vitamin $\mathrm{D}$ modulates many biological activities of the neural, immune and endocrine systems. The pleiotrophic characteristics of 1,25-dihydroxyvitamin $\mathrm{D}_{3}$-VDR complex are supported by the findings that VDR is expressed in at least 37 tissues, which the authors state can be roughly grouped into seven biological systems.

Due to the pleiotropic effect exerted by the 1,25-dihydroxyvitamin $\mathrm{D}_{3}-\mathrm{VDR}$ complex, genetic variants in $V D R$ have been found to be associated with a wide variety of diseases and phenotypes, including various cancers, asthma, calcium absorption, bone mineral density and hyperparathyroidism. They have also been linked to therapeutic responses to vitamin D and calcium supplements, antiresorptive treatments, calcipotriol and dexamethasone.
In their paper the authors describe two characteristic binding mechanisms of vitamin $\mathrm{D}_{3}$ to nuclear VDR (slow-acting genomic effect), and to VDR located in the caveolae in the plasma membrane (rapid response), further summarizing activated pathways.

Furthermore, pharmacogene summaries are needed to address significant associations to disease and therapeutic responses.

Source: Poon AH, Gong L, Brasch-Andersen $C$ et al. Very important pharmacogene summary for VDR. Pharmacogenet. Genomics doi:10.1097/FPC.0b013e328354455c (2012) (Epub ahead of print).

- All stories written by Hardaman Baryan 